\title{
Association of Plasmid-Mediated Quinolone Resistance with AmpC- Beta- Lactamase Producing E. coli strains from Different Sources
}

\author{
Norhan K Abd El-Aziz and Ahlam A Gharib \\ Department of Bacteriology, Mycology and Immunology, Faculty of Veterinary Medicine, \\ Zagazig University
} ABSTRACT

Objectives: this study was devoted to detect the plasmid-mediated quinolone resistance (PMQR) mechanisms ( $q n r$, qepA and $\left.a a c\left(6^{\prime}\right)-I b-c r\right)$ and its association with AmpC- Beta-lactamase
production in E. coli strains from different sources.

Methods: Twenty-nine $E$. coli isolates from food-producing animals (chicken and sheep) and their by-products, collected from Sharkia province, Egypt were tested for their susceptibilities for the presence of PMQR and AmpC $\beta$-lactamases genes in strains under study ser for screening Results: Out of 29 coli

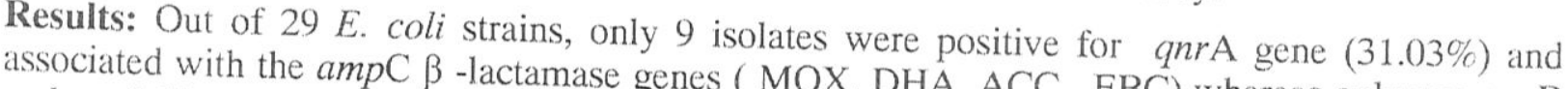
and qnrS-like genes $(3.45 \%)$ were detected. A qnr B , DHA, ACC, EBC) whereas only one qnrB $\mathrm{EBC}$ ) isolate. qepA and $a a c\left(6^{\prime}\right)-I b-c r$ were detected in $41 . \mathrm{r}$ well as qnrS was detected in (DHA, respectively alone or in combination with $q n r$ cted in $41.38 \%$ and $3.45 \%$ of the E.coli isolates, in $75.86 \%$ of all strains and in $100 \%$ and 66 renes. The ampC $\beta$-lactamase genes were detected strains, respectively, gave amplicons range f66 \% the PMQR determinant-positive and -negative electrophoresis.

Conclusions: A high prevalence of PMQR determinants among AmpC $\beta$-lactamase producing $E$. coli isolates from chicken mainly and their by-products was detected in Egypt. Their effect may slightly increase the MIC of quinolone and may related to the development of full resistance to
quinolone.

\section{INTRODUCTION}

In Egypt, quinolones and $\beta$-lactams are among the most commonly used antimicrobials in both human and veterinary clinical medicine. The widespread use of antibiotics in food animal production systems has resulted in the emergence of antibiotic resistant zoonotic bacteria that can be transmitted to humans through the food chain. Infection with antibiotic resistant bacteria negatively impacts on public health, due to an increased incidence of treatment failure and severity of disease (1).

Fluoroquinolone resistance is emerging in Gram-negative pathogens worldwide. Unfortunately, quinolone resistant $E$. coli in animals have increased in numbers after quinolone introduction (2). The traditional understanding that quinolone resistance is acquired only through mutation and transmitted only vertically does not entirely account for the relative ease with which resistance develops in exquisitely susceptible organisms, or for the very strong association between resistance to quinolones and to other agents. The recent discovery of plasmid-mediated horizontally transferable genes encoding quinolone resistance might shed light on these phenomena (3).

However, since the first plasmid-mediated quinolone resistance (PMQR) gene (qnrA) was 
reported in 1998 for a Klebsiella pneumoniae isolate from the United States (4), five different transferable mechanisms of quinolone resistance (TMQRs) have been described. These mechanisms including target protection (qnr genes), quinolone modification (aac $\left(6^{\prime}\right)$ $I b-c r)$, plasmid-encoded efflux systems (qepA or OqxAB, amongst others), effect on bacterial growth rates and natural transformation (5).

Qnr proteins belong to the pentapeptiderepeat family that directly protect DNA gyrase and topoisomerase IV from quinolone inhibition (6) leading to 8 to 32 -fold increase in MICs of quinolones (7). There are at least 6 qnrA, 20 qnrB, and 3 qnrS alleles descriped, with one or more amino acid alterations within each family (8), furthermore, $q n r C$ and $q n r D$ (one variant for each) were also reported $(9,10)$; a database of qnr allele designations are maintained at the website http://www.lahey.org/qnrStudies.

Qnr could bind to the gyrase holoenzyme and its respective subunits, GyrA and GyrB, and consequently topoisomerase IV and its subunits, ParC and ParE, such binding does not require the presence of the enzyme-DNAquinolone complex. It was hypothesized that the formation of Qnr-gyrase / Qnrtopoisomerase IV complex occurs before the formation of the cleavage complex. Furthermore, DNA binding by gyrase / topoisomerase IV decreases when gyrase / topoisomerase IV interacts with Qnr reducing the amount of holoenzyme-DNA targets for quinolone inhibition $(11,12)$.

$a a c\left(6^{\prime}\right)-I b-c r$ gene encodes a new variant of common aminoglycoside acetyltransferase. Two single amino acid substitutions, Trp102Arg and Asp179Tyr, in the wild-type allele $a a c\left(6^{\prime}\right)-I b$ enable the gene product to be capable of $\mathrm{N}$-acetylation of piperazinyl amine of certain fluoroquinolones and thereby reduces their antibacterial activities (13). It was first reported in 2003 and confers 2-4 folds increase in MICs (14).

The QepA determinant is an efflux pump protein putatively belonging to 14transmembrane-segment major facilitator superfamily of transporters involved in pumping of hydrophilic fluoroquinolones out of bacterial cells. It confers a 32-to 64-fold increase of fluoroquinolone MIC values. The novel qepA gene was identified on plasmid pHPA of Escherichia coli strain, which was isolated in 2002 from the urine of an inpatient in Japan; this plasmid displayed a multipleresistance profile for aminoglycosides, fluoroquinolones, and broad-spectrum $\beta$ lactams (15).

Today, emerging newer $\beta$-lactamase enzymes including extended-spectrum $\beta$ lactamases (ESBLs) and AmpC $\beta$-lactamases are associated with misuse of $\beta$-lactam antibiotics resulting in evolution of Beta-lactam resistance in Gram-negative bacteria, especially Escherichia coli (16).

AmpC $\beta$-lactamases belong to Ambler class $\mathrm{C}$ and, once expressed at high levels, confer resistance to a wide variety of $\beta$-lactam antibiotics including penicillins, most of the expanded spectrum cephalosporins, (excluding cefpirome and cefepime) and monobactams (17). Furthermore, AmpC enzymes, with only few exceptions, are not inhibited by the ESBL inhibitor clavulanic acid (18) and in a strain with decreased outer membrane permeability, such enzymes can provide resistance to carbapenems $(19,20)$.

Actually, AmpC $\beta$-lactamases can be either chromosomal or plasmid mediated. In E. coli, the natural chromosomal AmpC is constitutively produced at a very low level because of a transcriptional attenuator coupled with a weak promoter $(21,22)$. However, constitutive overexpression of AmpC can occur due to either the deregulation of the chromosomally encoded ampC gene (derepressed ampC mutants) or by acquisition of a transferable ampC gene, imported from the chromosomal genes, on a plasmid or other transferable elements (plasmid-mediated AmpC) conferring resistance similar to their chromosomal counterparts (23).

Six different groups of plasmid mediated AmpC were identified. These groups include ACC, DHA, CIT and EBC, which originated 
from $H$. alvei, M. morganii, C. freundii and $E$. cloacae, respectively, as well as FOX and MOX (unknown origins) (23). One important difference between $E$. coli and the other members of the family Enterobacteriaceae is that the expression of $a m p C$ in $E$. coli is not inducible (24).

Association of PMQR determinants with extended-spectrum beta-lactamases (ESBLs) or AmpC beta-lactamases is also noteworthy; as qnr genes were found to be carried on the same plasmid with various extended spectrum or AmpC-type $\beta$-lactamase genes (6).

Hence, the objective of this study was to determine the coexistence of PMQR determinants and AmpC beta-lactamases in $E$. coli isolates from livestock animals and their meat products in Egypt to assess their potential role as a reservoir of emerging multidrug resistant bacteria which may subsequently transmit to humans through food chain or human-animal interactions.

\section{MATERIAL AND METHODS}

Bacterial strains

E. coli isolates were collected from livestock animal farms (chicken and sheep) and their by-products from different sail shops; each isolate was from a separate animal. Twentynine $E$. coli isolates were collected, including 17 from respiratory organs or feacal samples from diseased food- producing animals (13 chickens and 4 sheep, respectively) and 12 isolates from their by-products in Sharkia province, Egypt. The by-product isolates were classified as 8 chicken by-products (4 of each chickens burger and luncheon) and 4 beef byproducts ( 2 burger and one of each sausage and minced meat). The bacterial strains were identified by classical biochemical methods and using rapid API20 E bacterial identification system (Biomerieux, France).

\section{Antimicrobial susceptibility testing}

Antimicrobial susceptibility testing was performed by Kirby-Bauer standard disk diffusion method onto Mueller-Hinton agar (Difco, USA), using overnight cultures at a 0.5 McFarland standard followed by incubation at $35^{\circ} \mathrm{C}$ for 16 to $18 \mathrm{~h}$ (25). The following antimicrobials were tested: nalidixic acid, ciprofloxacin, norfloxacin, levofloxacin, gatifloxacin, ampicillin, ampicillin-sulbactam, amoxycillin-clavulanic acid, piperacillin, imipenem, cefazolin, cefoxitin, cefuroxime, cefotaxime, ceftriaxone, cefepime, aztreonam. gentamicin, amikacin, erythromycin, tetracycline, chloramphenicol, colistin and sulfamethoxazole-trimethoprim (Oxoid, UK). Strains concomitantly resistant to $\geq 3$ antimicrobial classes were defined as multidrug-resistant (MDR).

Furthermore, the minimum inhibitory concentrations (MICs) of ciprofloxacin (Sigma, USA) were determined by reference broth microdilution method according to Clinical and Laboratory Standards Institute (26) guidelines using custom-designed 96-well panels (Corning, USA). The interpretive criteria were those published in the relevant CLSI document.

AmpC disc test

The test is based on use of Tris-EDTA to permeabilize a bacterial cell and release $\beta$ lactamases into the external environment. AmpC disks were prepared in-house by applying $20 \mu \mathrm{l}$ of a 1:1 mixture of saline and 100X Tris-EDTA (Sigma, USA) to sterile filter paper disks, allowing the disks to dry, and storing them at 2 to $8^{\circ} \mathrm{C}$. The surface of a Mueller-Hinton agar plate was inoculated with a lawn of cefoxitin susceptible E. coli strain according to the standard disk diffusion method (26). Immediately prior to use, AmpC disks were rehydrated with $20 \mu \mathrm{l}$ of saline and several colonies of each test organism were applied to a disk. A $30 \mu \mathrm{g}$ cefoxitin disk was placed on the inoculated surface of the Mueller-Hinton agar. The inoculated AmpC disk was then placed almost touching the antibiotic disk with the inoculated disk face in contact with the agar surface. The plate was inverted and incubated 
overnight at $35^{\circ} \mathrm{C}$ in ambient air. A flattening or indentation of the cefoxitin inhibition zone in the vicinity of the disk with test strain was interpreted as positive for the production of AmpC $\beta$-lactamase, while an undistorted zone was considered as negative (27).

Plasmid extraction

Thermo Scientific GeneJET Plasmid Miniprep Kit was used for plasmid DNA extraction from pelleted bacterial cell after harvesting on $1-5 \mathrm{ml} \mathrm{LP}$ media. The bacterial pellet was lysed and the plasmid DNA was bound on the silica membrane, which was then washed and eluted by $50 \mu \mathrm{l}$ of the elution buffer.
Detection of PMQR determinants and AmpC $\beta$ -lactamase-encoding genes

The isolates were investigated for the presence of qnrA, qnrB, qnrS, aac(6)-Ib-cr, qepA and AmpC $\beta$-lactamases genes by PCR amplification with the primer sets described in table (1). DNA samples were amplified in a total of $25 \mu \mathrm{l}$ of the following reaction mixture: $12.5 \mu \mathrm{l}$ DreamTaq TM Green Master Mix (2X) (Sigma, UK), $1 \mu$ of each primer (10pmole), $2 \mu \mathrm{l}$ template DNA and $8.5 \mu \mathrm{l}$ water nucleasefree. The amplified PCR product was electrophoresed on a 1.5\% agarose gel in Trisacetate-EDTA buffer. A 100-bp DNA ladder (Invitrogen, Carlsbad, CA) was used as a molecular weight marker.

Table 1. Oligonucleotide primers, amplification cycles and amplicons of PMQR and AmpC $\beta$-lactamases in $E$. coli strains

\begin{tabular}{|c|c|c|c|c|c|c|}
\hline \multirow[t]{2}{*}{ Genes } & \multirow{2}{*}{$\begin{array}{c}\text { Primers } \\
\text { "5-3" } \\
\end{array}$} & \multicolumn{3}{|c|}{ Amplification cycle } & \multirow{2}{*}{$\begin{array}{l}\text { Amplicons } \\
\text { (bp) }\end{array}$} & \multirow{2}{*}{ References } \\
\hline & & Denaturation & Annealing & Extension & & \\
\hline & $\begin{array}{l}\text { F: ATTTCTCACGCCAGGATTTG } \\
\text { R: GATCGGCAAAGGTTAGGTCA }\end{array}$ & & & & 516 & \\
\hline$q n r \mathrm{~B}$ & $\begin{array}{l}\text { F: GATCGTGAAAGCCAGAAAGG } \\
\text { R: ACGATGCCTGGTAGTTGTCC } \\
\text { F: ACGACATTCGTCAACTGCAA } \\
\text { R: TAAATTGGCACCCTGTAGGC }\end{array}$ & $94^{\circ} \mathrm{C} 45 \mathrm{~S}$. & $53^{\circ} \mathrm{C} 45 \mathrm{~S}$. & $72^{\circ} \mathrm{C} 45 \mathrm{~S}$ & 417 & (1.3) \\
\hline$a a c\left(6^{\prime}\right)-I b-c r$ & $\begin{array}{l}\text { F: CCCGCTTTCTCGTAGCA } \\
\text { R: TTAGGCATCACTGCGTCTTC }\end{array}$ & $94^{\circ} \mathrm{C} 30 \mathrm{~S}$ & $52^{\circ} \mathrm{C} 30 \mathrm{~S}$ & $72^{\circ} \mathrm{C} 30 \mathrm{~S}$ & 113 & (28) \\
\hline $\begin{array}{c}q e p A \\
\text { MoXM }\end{array}$ & $\begin{array}{l}\text { F: CGTGTTGCTGGAGTTCTTC } \\
\text { R: CTGCAGGTACTGCGTCATG }\end{array}$ & $94^{\circ} \mathrm{C} 45 \mathrm{~S}$. & $50^{\circ} \mathrm{C} 45 \mathrm{~S}$ & $72^{\circ} \mathrm{C} 45 \mathrm{~S}$ & 403 & (29) \\
\hline $\begin{array}{c}\text { (MOX-1, MOX-2, } \\
\text { CMY-1, CMY-8 to } \\
\text { CMY-11) }\end{array}$ & $\begin{array}{l}\text { F: GCTGCTCAAGGAGCACAGGAT } \\
\text { R:CACATTGACATAGGTGTGGTGC }\end{array}$ & & & & 520 & \\
\hline $\begin{array}{c}\text { CITM } \\
\text { (LAT-1 to LAT-4, } \\
\text { CMY-2 to CMY-7, } \\
\text { BIL-1) }\end{array}$ & $\begin{array}{l}\text { F: TGGCCAGAACTGACAGGCAAA } \\
\text { R: TTTCTCCTGAACGTGGCTGGC }\end{array}$ & $94^{\circ} \mathrm{C} 45 \mathrm{~S}$. & $55^{\circ} \mathrm{C} 45 \mathrm{~S}$ & $72^{\circ} \mathrm{C} 45 \mathrm{~S}$ & 462 & \\
\hline $\begin{array}{c}\text { DHAM } \\
\text { (DHA-1, DHA-2) }\end{array}$ & $\begin{array}{l}\text { F: AACTTTCACAGGTGTGCTGGGT } \\
\text { R: CCGTACGCATACTGGCTTTGC }\end{array}$ & & & & 405 & (23) \\
\hline $\begin{array}{l}\mathrm{ACCM} \\
(\mathrm{ACC})\end{array}$ & $\begin{array}{l}\text { F: AACAGCCTCAGCAGCCGGTTA } \\
\text { R: TTCGCCGCAATCATCCCTAGC }\end{array}$ & & & & 346 & \\
\hline EBCM & F: TCGGTAAAGCCGATGTTGCGG & & & & & \\
\hline (MIR-1T ACT-1) & R: CTTCCACTGCGGCTGCCAGTT & & & & 302 & \\
\hline $\begin{array}{c}\text { FOXM } \\
(\mathrm{FOX}-1 \text { to FOX-5b) } \\
\end{array}$ & $\begin{array}{l}\text { F:AACATGGGGTATCAGGGAGATG } \\
\text { R: CAAAGCGCGTAACCGGATTGG }\end{array}$ & $94^{\circ} \mathrm{C} 30 \mathrm{~S}$. & $53^{\circ} \mathrm{C} 30 \mathrm{~S}$ & $72^{\circ} \mathrm{C} 45 \mathrm{~S}$ & 190 & \\
\hline
\end{tabular}




\section{RESULTS}

Antimicrobial susceptibility testing

E. coli strains were tested against 24 antimicrobial agents including in 11 different groups (Table 2). The results showed that $82.76 \%$ of the isolates were resistant to nalidixic acid, additionally; high level of resistance was recorded for fluoroquinolones tested as ciprofloxacin (72.4\%), norfloxacin and levofloxacin (62\%) and gatifloxacin $(51.7 \%)$. Regarding to beta-lactams, absolute resistance was detected for ampicillin, amoxycillin-clavulanic acid, piperacillin and cefazolin meanwhile, $79.3 \%$ of the isolates were resistant to cefuroxime, $72.4 \%$ to aztreonam, $69 \%$ to cefepime and $65.5 \%$ to cefoxitin, cefotaxime, ceftriaxone, and low level of resistance was reported for imipenem $(3.4 \%)$. Furthermore, all tested E. coli strains were considered as multidrug resistant. It is noteworthy that all $E$. coli strains were sensitive to amikacin, which is considered as a drug of choice for treatment (Table 2).

Table 2. Antibiogram for $E$. coli strains using disk diffusion test

\begin{tabular}{|c|c|c|c|c|c|c|c|c|c|c|c|c|c|c|c|c|c|c|c|c|c|c|c|c|}
\hline Strain No & $\frac{1}{z}$ & $\frac{x}{0}$ & $\frac{1}{z}$ & $\frac{x}{2}$ & $\frac{X}{0}$ & $\sum$ & $\sum_{j}$ & $\sum_{n}$ & 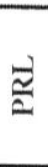 & 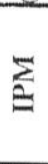 & U & 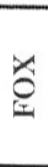 & $\sum$ & $\underline{u}$ & $\underset{\widetilde{U}}{\cong}$ & 空 & $\bar{\alpha}$ & Z & 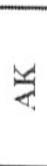 & $u$ & 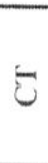 & $E$ & $(-1)$ & $=$ \\
\hline 1 & $\mathrm{R}$ & $\mathrm{R}$ & $\mathrm{R}$ & $\mathrm{R}$ & $\mathrm{R}$ & $R$ & $\mathrm{R}$ & $\mathrm{R}$ & $\mathrm{R}$ & S & $\mathrm{R}$ & $\mathrm{R}$ & $\mathrm{R}$ & $\mathrm{R}$ & I & $\mathrm{S}$ & $R$ & $\mathrm{~S}$ & $\mathrm{~S}$ & I & $\mathrm{S}$ & $\mathrm{R}$ & $\mathrm{R}$ & R \\
\hline 2 & $\mathrm{R}$ & $\mathrm{R}$ & $\mathrm{R}$ & I & $\mathrm{R}$ & $\mathrm{R}$ & $\mathrm{R}$ & S & $\mathrm{R}$ & S & $\mathrm{R}$ & $\mathrm{R}$ & $\mathrm{R}$ & $\mathrm{R}$ & S & $\mathrm{R}$ & $\mathrm{R}$ & S & S & $\mathrm{R}$ & $S$ & I & $\mathrm{R}$ & $\mathrm{R}$ \\
\hline 3 & $\mathrm{R}$ & S & S & S & S & $\mathrm{R}$ & $\mathrm{R}$ & I & $\mathrm{R}$ & S & $\mathrm{R}$ & S & $\mathrm{R}$ & $\mathrm{R}$ & I & $\mathrm{R}$ & $\mathrm{R}$ & S & S & $\mathrm{S}$ & S & $\mathrm{R}$ & R & S \\
\hline 4 & $\mathrm{R}$ & $\mathrm{R}$ & $\mathrm{R}$ & $\mathrm{R}$ & I & $\mathrm{R}$ & $\mathrm{R}$ & $\mathrm{R}$ & $\mathrm{R}$ & $\mathrm{S}$ & $\mathrm{R}$ & S & $\mathrm{R}$ & $\mathrm{R}$ & $\mathrm{R}$ & I & $\mathrm{R}$ & S & S & I & S & $\mathrm{R}$ & $\mathrm{R}$ & $\mathrm{R}$ \\
\hline 5 & $\mathrm{R}$ & $\mathrm{R}$ & $\mathrm{R}$ & $\mathrm{R}$ & $\mathrm{R}$ & $\mathrm{R}$ & $\mathrm{R}$ & $\mathrm{R}$ & $\mathrm{R}$ & S & $\mathrm{R}$ & $\mathrm{R}$ & $\mathrm{R}$ & $\mathrm{R}$ & S & $\mathrm{R}$ & $\mathrm{R}$ & S & S & $\mathrm{R}$ & S & $\mathrm{R}$ & $\mathrm{R}$ & R \\
\hline 6 & R & $\mathrm{R}$ & $\mathrm{R}$ & $\mathrm{R}$ & $\mathrm{R}$ & $\mathrm{R}$ & $\mathrm{R}$ & I & $\mathrm{R}$ & S & $\mathrm{R}$ & $\mathrm{R}$ & I & S & I & S & $\mathrm{R}$ & R & S & $\mathrm{R}$ & S & $\mathrm{R}$ & $\mathrm{R}$ & S \\
\hline 7 & $\mathrm{R}$ & $\mathrm{R}$ & $\mathrm{R}$ & $\mathrm{R}$ & $\mathrm{R}$ & $\mathrm{R}$ & $\mathrm{R}$ & $\mathrm{R}$ & $\mathrm{R}$ & $S$ & $\mathrm{R}$ & $S$ & $S$ & S & $\mathrm{R}$ & S & $\mathrm{R}$ & $S$ & S & $\mathrm{R}$ & $S$ & $\mathrm{R}$ & $\mathrm{R}$ & $\mathrm{R}$ \\
\hline 8 & $\mathrm{R}$ & $\mathrm{R}$ & $\mathrm{R}$ & $\mathrm{R}$ & $\mathrm{R}$ & $\mathrm{R}$ & $\mathrm{R}$ & I & $\mathrm{R}$ & $S$ & $\mathrm{R}$ & S & $\mathrm{R}$ & S & I & S & $\mathrm{R}$ & S & S & $\mathrm{R}$ & $\mathrm{S}$ & $\mathrm{R}$ & $\mathrm{R}$ & $\mathrm{R}$ \\
\hline 9 & $\mathrm{R}$ & $\mathrm{R}$ & $\mathrm{R}$ & $\mathrm{R}$ & $\mathrm{R}$ & $\mathrm{R}$ & $\mathrm{R}$ & $\mathrm{R}$ & $\mathrm{R}$ & $S$ & $\mathrm{R}$ & S & $\mathrm{S}$ & $S$ & I & S & $\mathrm{R}$ & $\mathrm{R}$ & S & $\mathrm{R}$ & $S$ & $\mathrm{R}$ & $\mathrm{R}$ & $\mathrm{S}$ \\
\hline 10 & $\mathrm{R}$ & $\mathrm{S}$ & $\mathrm{S}$ & $\mathrm{S}$ & $\mathrm{S}$ & $\mathrm{R}$ & $\mathrm{R}$ & $S$ & $\mathrm{R}$ & S & $\mathrm{R}$ & $S$ & S & $\mathrm{S}$ & S & S & $\mathrm{R}$ & S & S & S & S & $\mathrm{R}$ & $\mathrm{R}$ & $\mathrm{R}$ \\
\hline 11 & $\mathrm{R}$ & $\mathrm{R}$ & $\mathrm{R}$ & $\mathrm{R}$ & $\mathrm{R}$ & $\mathrm{R}$ & $\mathrm{R}$ & $\mathrm{R}$ & $\mathrm{R}$ & $S$ & $\mathrm{R}$ & S & $\mathrm{R}$ & $\mathrm{R}$ & $\mathrm{R}$ & $\mathrm{R}$ & $\mathrm{R}$ & $\mathrm{R}$ & S & I & $\mathrm{S}$ & $\mathrm{R}$ & $\mathrm{R}$ & $\mathrm{R}$ \\
\hline 12 & $\mathrm{R}$ & $\mathrm{R}$ & $\mathrm{R}$ & $\mathrm{R}$ & $\mathrm{R}$ & $\mathrm{R}$ & $\mathrm{R}$ & $\mathrm{R}$ & $\mathrm{R}$ & $S$ & $\mathrm{R}$ & S & $\mathrm{S}$ & S & $\mathrm{R}$ & $\mathrm{R}$ & $\mathrm{R}$ & $\mathrm{R}$ & S & I & $S$ & $\mathrm{R}$ & $\mathrm{R}$ & $\mathrm{S}$ \\
\hline 13 & $\mathrm{R}$ & $\mathrm{S}$ & $\mathrm{S}$ & $\mathrm{S}$ & $\mathrm{S}$ & $\mathrm{R}$ & $\mathrm{R}$ & $\mathrm{R}$ & $\mathrm{R}$ & $S$ & $\mathrm{R}$ & $\mathrm{S}$ & $S$ & $S$ & I & $\mathrm{R}$ & $\mathrm{R}$ & S & $\mathrm{S}$ & $S$ & S & $\mathrm{R}$ & $\mathrm{R}$ & $\mathrm{R}$ \\
\hline 14 & $\mathrm{R}$ & $\mathrm{R}$ & $\mathrm{R}$ & $\mathrm{R}$ & $\mathrm{R}$ & $\mathrm{R}$ & $\mathrm{R}$ & $\mathrm{R}$ & $\mathrm{R}$ & $S$ & $\mathrm{R}$ & $\mathrm{R}$ & $\mathrm{R}$ & $\mathrm{R}$ & $\mathrm{R}$ & I & $\mathrm{S}$ & $\mathrm{R}$ & $\mathrm{S}$ & $\mathrm{R}$ & $\mathrm{R}$ & $\mathrm{R}$ & $\mathrm{R}$ & $\mathrm{R}$ \\
\hline 15 & $\mathrm{R}$ & $\mathrm{R}$ & $\mathrm{R}$ & I & $\mathrm{R}$ & $\mathrm{R}$ & $\mathrm{R}$ & $\mathrm{R}$ & $\mathrm{R}$ & $S$ & $\mathrm{R}$ & I & $\mathrm{R}$ & $\mathrm{R}$ & $\mathrm{R}$ & $\mathrm{R}$ & I & $\mathrm{R}$ & $\mathrm{S}$ & $\mathrm{R}$ & $\mathrm{S}$ & $\mathrm{R}$ & $\mathrm{R}$ & $\mathrm{R}$ \\
\hline 16 & $\mathrm{R}$ & $\mathrm{R}$ & $\mathrm{R}$ & $\mathrm{R}$ & $\mathrm{S}$ & $\mathrm{R}$ & $\mathrm{R}$ & $\mathrm{R}$ & $\mathrm{R}$ & $S$ & $\mathrm{R}$ & I & $\mathrm{R}$ & $\mathrm{R}$ & $\mathrm{R}$ & $\mathrm{S}$ & $\mathrm{R}$ & $\mathrm{R}$ & $\mathrm{S}$ & $\mathrm{R}$ & $\mathrm{R}$ & $\mathrm{R}$ & $\mathrm{R}$ & $\mathrm{R}$ \\
\hline 17 & $\mathrm{R}$ & S & $\mathrm{R}$ & $\mathrm{R}$ & $\mathrm{R}$ & $\mathrm{R}$ & $\mathrm{R}$ & $\mathrm{R}$ & $\mathrm{R}$ & $S$ & $\mathrm{R}$ & $\mathrm{R}$ & $\mathrm{R}$ & $\mathrm{R}$ & $\mathrm{R}$ & S & $\mathrm{R}$ & I & $\mathrm{S}$ & $\mathrm{R}$ & S & $\mathrm{R}$ & $\mathrm{R}$ & $\mathrm{R}$ \\
\hline 18 & $\mathrm{R}$ & $\mathrm{R}$ & $\mathrm{R}$ & $\mathrm{R}$ & $\mathrm{R}$ & $\mathrm{R}$ & $\mathrm{R}$ & $\mathrm{R}$ & $\mathrm{R}$ & $\mathrm{S}$ & $\mathrm{R}$ & $\mathrm{R}$ & $\mathrm{R}$ & $\mathrm{R}$ & $\mathrm{R}$ & S & $\mathrm{R}$ & $\mathrm{R}$ & $S$ & $\mathrm{R}$ & S & $\mathrm{R}$ & $\mathrm{R}$ & $\mathrm{R}$ \\
\hline 19 & $\mathrm{R}$ & $\mathrm{R}$ & $\mathrm{R}$ & $\mathrm{R}$ & $\mathrm{S}$ & $\mathrm{R}$ & $\mathrm{R}$ & $\mathrm{R}$ & $\mathrm{R}$ & S & $\mathrm{R}$ & $\mathrm{R}$ & $\mathrm{R}$ & $\mathrm{R}$ & $\mathrm{R}$ & $\mathrm{R}$ & $\mathrm{R}$ & $\mathrm{R}$ & $\mathrm{S}$ & $\mathrm{R}$ & $\mathrm{R}$ & $\mathrm{R}$ & $\mathrm{R}$ & $\mathrm{R}$ \\
\hline 20 & $\mathrm{R}$ & $\mathrm{R}$ & I & I & $\mathrm{S}$ & $\mathrm{R}$ & $\mathrm{R}$ & $\mathrm{R}$ & $\mathrm{R}$ & S & $\mathrm{R}$ & $\mathrm{R}$ & $\mathrm{R}$ & $\mathrm{R}$ & $\mathrm{R}$ & $\mathrm{R}$ & $\mathrm{R}$ & S & $\mathrm{S}$ & $\mathrm{R}$ & $\mathrm{R}$ & S & $\mathrm{R}$ & $\mathrm{R}$ \\
\hline 21 & $\mathrm{R}$ & $\mathrm{R}$ & $\mathrm{S}$ & $\mathrm{S}$ & $S$ & $\mathrm{R}$ & $\mathrm{R}$ & $\mathrm{R}$ & $\mathrm{R}$ & $S$ & $\mathrm{R}$ & $\mathrm{R}$ & $\mathrm{R}$ & $\mathrm{R}$ & $\mathrm{R}$ & S & $\mathrm{R}$ & S & $S$ & $\mathrm{R}$ & $\mathrm{R}$ & S & $\mathrm{R}$ & $\mathrm{R}$ \\
\hline 22 & $\mathrm{~S}$ & $\mathrm{~S}$ & $\mathrm{~S}$ & S & $\mathrm{S}$ & $\mathrm{R}$ & $\mathrm{R}$ & $S$ & I & $S$ & $R$ & $\mathrm{R}$ & $\mathrm{R}$ & $R$ & S & $\mathrm{R}$ & $S$ & S & $S$ & $S$ & $\mathrm{R}$ & $\mathrm{S}$ & $\mathrm{R}$ & $\mathrm{R}$ \\
\hline 23 & $\mathrm{~S}$ & $\mathrm{~S}$ & $\mathrm{~S}$ & $\mathrm{~S}$ & $\mathrm{~S}$ & $R$ & $R$ & I & I & $\mathrm{S}$ & $\mathrm{R}$ & $\mathrm{S}$ & $\mathrm{S}$ & $\mathrm{R}$ & S & S & S & S & $S$ & $S$ & $S$ & $\mathrm{~S}$ & $\mathrm{R}$ & $S$ \\
\hline 24 & $\mathrm{~S}$ & $\mathrm{~S}$ & $S$ & $\mathrm{~S}$ & $\mathrm{~S}$ & $\mathrm{R}$ & I & $S$ & $\mathrm{R}$ & $S$ & $\mathrm{R}$ & $\mathrm{R}$ & $\mathrm{R}$ & $\mathrm{R}$ & S & $S$ & $\mathrm{~S}$ & S & $\mathrm{S}$ & $S$ & $S$ & $\mathrm{~S}$ & $\mathrm{R}$ & I \\
\hline 25 & $S$ & S & $S$ & $S$ & $S$ & $\mathrm{R}$ & $\mathrm{R}$ & $S$ & I & $S$ & $\mathrm{R}$ & $\mathrm{R}$ & $\mathrm{R}$ & $\mathrm{R}$ & S & $S$ & S & S & $S$ & S & $S$ & $S$ & $\mathrm{R}$ & $S$ \\
\hline 26 & $\mathrm{~S}$ & S & $\mathrm{S}$ & S & $S$ & $\mathrm{R}$ & $\mathrm{R}$ & $\mathrm{R}$ & $\mathrm{R}$ & S & $\mathrm{R}$ & $\mathrm{R}$ & $\mathrm{R}$ & S & S & S & $\mathrm{R}$ & S & $S$ & $S$ & S & $\mathrm{R}$ & $\mathrm{R}$ & $S$ \\
\hline 27 & R & R & $S$ & S & S & $\mathrm{R}$ & $\mathrm{R}$ & $S$ & $\mathrm{R}$ & $S$ & $\mathrm{R}$ & $\mathrm{R}$ & $\mathrm{R}$ & $\mathrm{S}$ & S & $S$ & $\mathrm{R}$ & $\mathrm{R}$ & $S$ & S & S & $\mathrm{R}$ & $\mathrm{R}$ & I \\
\hline 28 & R & $\mathrm{S}$ & $\mathrm{S}$ & S & $S$ & $\mathrm{R}$ & $\mathrm{R}$ & I & $\mathrm{S}$ & S & $\mathrm{R}$ & $\mathrm{R}$ & R & S & S & S & $S$ & $\mathrm{~S}$ & $S$ & S & S & $\mathrm{S}$ & $\mathrm{R}$ & S \\
\hline 29 & R & $\mathrm{R}$ & $\mathrm{R}$ & $\mathrm{R}$ & $\mathrm{R}$ & $\mathrm{R}$ & $\mathrm{R}$ & $\mathrm{R}$ & $\mathrm{R}$ & $\mathrm{R}$ & $\mathrm{R}$ & I & $\mathrm{R}$ & $\mathrm{R}$ & $\mathrm{R}$ & $S$ & $\mathrm{R}$ & $\mathrm{R}$ & $S$ & $\mathrm{R}$ & $\mathrm{S}$ & $\mathrm{R}$ & $\mathrm{R}$ & $\mathrm{R}$ \\
\hline
\end{tabular}

AMA: Antimicrobial agent, NAL: nalidixic acid, CPFX: ciprofloxacin, NFLX: norfloxacin, I.VFX: levofloxacin, GFLX: gatifloxacin, AM: ampicillin, AMC: amoxycillin-clavulanic acid, SAM: ampicillin-sulbactam, PRI: piperacillin, IPM: imipenem, CZ: cefazolin, FOX: cefoxitin, CXM: cefuroxime, CTX: cefotaxime, CRO: ceftriaxone, FEB: cefepime, SXT: sulfamethoxazole-trimethoprim, CN: gentamicin, AK: amikacin, C: chloramphenicol, CT: colistin TE: tetracycline, E: erythromycin, ATM: aztreonam, R: resistant, S: sensitive, I: intermediate 


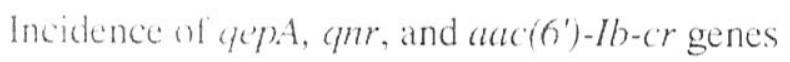

Among the total of $29 \mathrm{E}$. coli strains, PMQR determinants were present in $48.27 \%$, with qepA, qnr, and aac $\left(6^{\prime}\right)-I b-c r$ being detected alone or in combination in $41.38 \%$, $31.03 \%$, and $3.45 \%$ of the strains, respectively. The anr genes included 9 qnrA, and one of both qurl and ymrs. One strain was positive for aace(6')-Ih-cr or qurb or qurs in addition to (c) A, while seven strains harbored both qurA and qepA. qnrA, qnrB, and qepA coexisted in a strain of $E$. coli isolated from respiratory organs of chicken (No.6) as well as qurA, qurs, and qepA coexisted in a strain of $E$. coli isolated from chicken burger (No.14). Detailed information on these PMQR determinantpositive isolates is given in Table (3) and Fig (1). Among the 17 isolates from livestock animals. $64.7 \%$ strains of chicken origin only carried at least one PMOR determinant; $47.6 \%$. $5.88 \%$ and $52.9 \%$ strains were positive for $q n r$ genes, aac $\left.\left(f^{\prime}\right)-I /\right)-c r$, and qepA, respectively. Sheep feacal strains were not showed any PMQR determinants. Of the 12 isolates from animals by-products, $25 \%$ contained one or more PMQR determinants: $25 \%$ strains were positive for qepA genes (2 of sheep origin and one of chicken origin), only one of them (chicken origin) was positive for both qurA and quris (No.14). qnrB and auc(6')-Ib-cr are not detected.

The incidence of PMQR determinants was significantly higher in livestock animal strains
$(64.7 \%)$ than in by-products strains $(25 \%)$. The difference contributed to the higher incidence of qurA and qepA in animals than in their byproducts.

Identification of plasmid-mediated AmpC $\beta$ lactamases in E. coli strains

AmpC $\beta$-lactamases were detected genotypically by PCR in 75. S6 of of all strains and in $100 \%$ and $66.66 \%$ of the PMQR determinant-positive and -negative strains, respectively. Among the ampC genes detected in AmpC-positive strains, twenty-one had EBC $\beta$-lactamase gene, eighteen had MOX gene. fifteen had DHA gene, nine had ACC gene and three had the CIT $\beta$-lactamase gene. showing amplicon sizes of $3(1) 2 \mathrm{bp}, 52() \mathrm{bp}, 4(15 \mathrm{bp}, 34 \mathrm{~h}$ bp and 462 bp respectively (Fig 2), while FOX $\beta$-lactamases gene was not detected in any of them. The $\mathrm{AmpC} \beta$-lactamases present in all 14 strains positive for PMOR determinants are listed in Table (3). PMQR determinants were detected in $63.63 \%$ of 22 isolates positive for AmpC $\beta$-lactamases that reflect multidrug resistance within the strains and strong association between gnr genes and plasmid carrying amp $\mathrm{C}$ genes.

The AmpC $\beta$-lactamases were detected phenotypically in 8 strains $(57.1 \%$ ) (of 14 ampC-PCR positive strains) only, ats a flattening or an indentation the zonce of inhibition indicating enzymatic inactivation of cefoxitin as shown in Fig (3). 


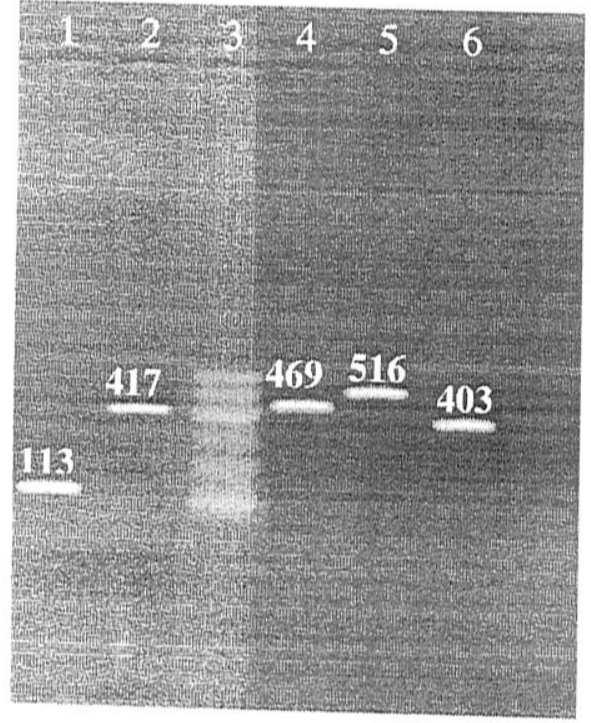

Fig 1. Agarose gel electrophoresis representing amplicons of PMQR determinants in base pairs. Lane 1 : (alac(6)-Ih-cr, lane 2: qnrS, lane 3: molecular size markers (100 bp), lane 4: qnrB, lane 5 qurA and lane 6:

Fig 3. AmpC disc test result showing flattening or an indentation the zone of inhibition indicating enzymatic inactivation of cefoxitin.

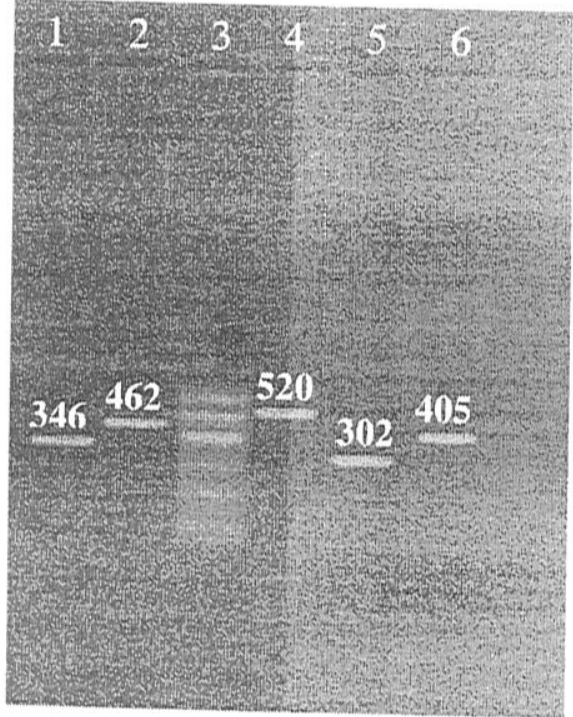

Fig 2. Agarose gel electrophoresis representing amplicons of $\mathrm{AmpC}$ $\beta$-lactamase genes in base pairs. Lane 1: $A C C$, lane 2: CIT, lane 3: molecular size markers (l(1)( ) bp). lane 4: $M O X$, lane $5 E B C$ and lane 6: DHA

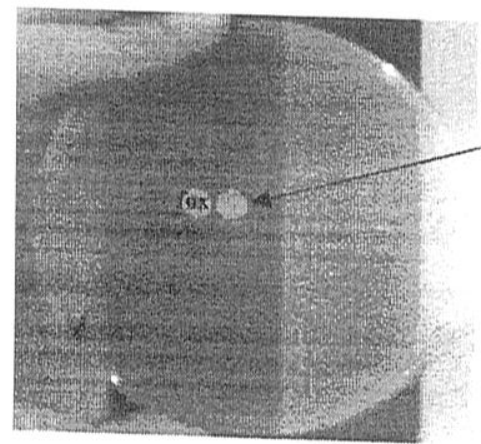


Table 3. Characteristics of PMQR determinants and AmpC $\beta$-lactamase of Ecoli strains recovered from different sources

\begin{tabular}{|c|c|c|c|c|c|}
\hline $\begin{array}{l}\text { Strain } \\
\text { No. }\end{array}$ & Animal source & Origin & $\begin{array}{c}\text { CFLX MIC } \\
\mu \mathrm{g} / \mathrm{ml}\end{array}$ & $\begin{array}{c}\text { PMQR } \\
\text { determinant }\end{array}$ & AmpC $\beta$-lactamase \\
\hline 1 & $C F$ & RO & 128 & qnirA, qepA & MOX, DHA, ACC,EBC \\
\hline 2 & $\mathrm{CF}$ & $\mathrm{RO}$ & 256 & qepA & $M O X, D H A, A C C, E B C$ \\
\hline 3 & $\mathrm{CF}$ & RO & 32 & ND & $M() X, D H A, A C C, E B C$ \\
\hline 4 & $\mathrm{CF}$ & RO & 1024 & qura, qepA & $M O X, D H A, A C C, E B C$ \\
\hline 5 & $\mathrm{CF}$ & RO & 256 & qnrA. qepA & $M O X, D H A, A C C, E B C$ \\
\hline 6 & $\mathrm{CF}$ & RO & 256 & $q n r A, q n r B, q e p A$ & DHA, EBC \\
\hline 7 & $C F$ & RO & 1024 & qnrA, qерA & $M O X, D H A, A C C, E B C$ \\
\hline 8 & CF & $\mathrm{RO}$ & 1024 & QepA, aac (6')-Ib-cr & $\mathrm{EBC}$ \\
\hline 9 & $\mathrm{CF}$ & $\mathrm{RO}$ & 1024 & qерA & $M O X, D H A, A C C, E B C$ \\
\hline 10 & CF & RO & 1024 & $N D$ & $M O X, D H A, E B C$ \\
\hline 11 & $\mathrm{CF}$ & RO & 1024 & qurA & $M O X, I) H A, A C C, F B C$ \\
\hline 12 & $\mathrm{CH}$ & RO & 1024 & qurA & $M() X, I) H A, I B C$ \\
\hline 13 & $\mathrm{CF}$ & RO & 16 & qurA, qepA & $M O X, D H A E B C$ \\
\hline 14 & $(-b p$ & $\mathrm{CB}$ & 16 & qnrA, qnrS. qepd & $D H A, E B C$ \\
\hline 15 & ( - bp & $\mathrm{CB}$ & 1024 & ND & $D H A, E B C$ \\
\hline 16 & C.lop & $\mathrm{CB}$ & 16 & $N D$ & ND \\
\hline 17 & C-bp & $\mathrm{CB}$ & 8 & $N D$ & MOX, EBC \\
\hline 18 & C-bp & $\mathrm{CL}$ & 1024 & $N D$ & MOX, EBC \\
\hline 19 & C-bp & $\mathrm{CL}$ & 32 & $N D$ & MOX, CIT, EBC \\
\hline 20 & C-bp & $\mathrm{CL}$ & 512 & qepA & $M O X, C I T, D H A, A C C, E B C$ \\
\hline 21 & C-bp & $\mathrm{CL}$ & 1024 & qepA & MOX, CIT \\
\hline 22 & A-bp & $\mathrm{BB}$ & 1 & $N D$ & ND \\
\hline 23 & A-bp & $\mathrm{BB}$ & 0.5 & $N D$ & ND \\
\hline 24 & A-bp & BS & 1 & $N D$ & ND \\
\hline 25 & A-bp & MM & 0.25 & $N D$ & ND \\
\hline 26 & SF & feces & 2 & $N D$ & MOX, EBC \\
\hline 27 & SF & feces & 1024 & $N D$ & MOX, EBC \\
\hline 28 & SF & feces & 1 & $N D$ & ND \\
\hline 29 & SF & feces & 32 & $N D$ & ND \\
\hline
\end{tabular}

CF: chicken farm, C-bp: chicken by-product, A-bp: animal by products, $\mathrm{SF}$ : sheep farms, RO; respiratory organs, $\mathrm{CB}$ : Chicken burger. (L: chicken luncheon, BB: beef burger, BS: , beef sausage, MM: minced meat,
NI): nnt detected

\section{DISCUSSION}

In the present study, the rates of quinolone resistance in $L$. coli strains were high in Egypt; more than 5()$\%$ of $E$. coli strains were resistant (1) quinolones and fluoroquinolones especially in strains producing AmpC beta-lactamase which was in accordance with (30).
Plasmid mediated quinolone resistant determinents were highly prevalent $(48.27$ \% $)$ in E. coli isolates from different sources in Egypt and in these strains, qepA and qniA were more common than other determinants. This percentage was higher than that $(34.7 \%$ ) of enterobacteriaceate isolates (mainly $E_{\text {. }}$ coli and $K$. pneumonices) of animals from Chind, that 
included mostly qepA and aac(6')-Ib-cr genes (31). Among 29 E. coli strains, qnr being detected alone or in combination with qepA and aac $\left(6^{\prime}\right)-I b-c r$ genes in $9(31.03 \%)$ isolates and included mainly anrA, and only one of both ynr $B$ and ynrs. Different result was obtained by (3) in United States that. ynr genes were signilicantly more prevalent in Enterohacter species $(31 \%)$ and $K$. pneumoniae $(20 \%)$ isolates than in E. coli isolates $(4 \%)$ with equivalent frequencies for qnrA and qnrB while qur:S wats absent. Also, (3I) in China recorded that ynr genes were prevalent in $7.9 \%$ of $F$. coll iscolates and were mainly gnrB and gnrs.

The ynr:S as well as alac( $\left.f^{\prime}\right)$-Ih-cr gene were previously found in $E$. coli isolates from China by (32) in pig. (3.3) in poultry and swine and (3I) in poultry and pig. All of qnr positive isolites showed decreased susceptibility to fluoroquinolones, mainly ciprofloxacine (Table 3).

A yep A is the most common than other determinants in this study, however the prevalence of qepA was low $(0.3 \%)$ in $E$. coli clinical isolates collected previously from 140) Japranesc hospitals in (34)

In currenl report, about $75.86 \%$ isolates of 1. coli carried plasmid-mediated AmpC $\beta$ lactamase genes by PCR and $63.63 \%$ of them revealed PMQR determinants. This linkage between conr determinants and AmpC Blactamases was described in several reports as cumpR gene which regulates the expression of ampl may be present between qur and the $3^{\circ}$ (S (quCE!l and sull) or is replaced by qnr, which may in turn explain it $(14,35)$

Moreover. a qur A gene was associated with the AmpC (MOX. DHA, ACC, EBC) in nine strains and the data has not been recently reported. Also, a qur B gene was found to be associated with the AmpC $\beta$-lactamase (DHA and EBC) (strains No.6). Simillarly, the association between qnrB4 variant and plasmid-mediated AmpC DHA-1has been previously detected in E. coli and K.pnemmoniae clinical isolates $(31,36)$. Additionally: qurs genes in strain No. 14 was associaled with the AmpC $\beta$-lactamase (DHA and $E B C)$, similar data was recorded previously by $(37,38)$ while not recorded by $(31)$.

Eight E. coli $(57.1 \%)$ only out of 14 (ampC -PCR positive) strains were positive for phenotypic confirmation of ampC phenotype that reflect accuracy of PCR as genotypic detection more than phenotypic detection and there is no standard phenotypic method for detection of such enzymes. Similar results was decided by CLSI.

Finally, our study showed a high prevalence of PMQR determinants among AmpC producing $E$. coli isolates that reflect multidrug resistance within the strains and strong association between qur genes and plasmid carrying ampC genes. The high prevalence of PMQR determinants and or AmpC $\beta$ lactamases in isolates from livestock animals may related to extensive use of broad spectrum antimicrobial agents resulting in spread and increase detection of these resistance determinents among bacteria of animal origin then of human origin which is important to the public health concern.

\section{REFERENCES}

1. Walsh C and Fanning S (2008): Antimicrobial resistance in food borne pathogens a cause for concern? Curr Drug Targets. $9(9): 8() \$-815$.

2.Webber $M$ and Piddock L. J V (2001): Quinolone resistance in Escherichica coli. Vet. Res. 32, 275-284.

3.Robicsek A, Strahilevitz. J, Sahm D F, Jacoby $G A$ and Hooper D C (2006a): qnir prevalence in ceftazidime-resistant Enterobacteriaceat isolates from the United States. Antimicrob. Agents Chemother. 50: 28722874 . 
4.Martinez-Martinez L, Pascual A and Jacoby $G A$ (1998): Quinolone resistance from a transferable plasmid. Lancet; 351:797-799.

5.Joaquim Ruiz, Maria $J P$ and Claudia $G$ (2012): Transferable mechanisms of quinolone resistance. International Journal of Antimicrobial Agents, 40: 196-203.

6.Robicsek A, Jacoby $G A$, and Hooper D C (2006b): The worldwide emergence of plasmid-mediated quinolone resistance. Lancet Infect. Dis. 6:629-640.

7.Tran $J H$, and Jacoby $G$ A (2002): Mechanism of plasmid-mediated quinolone resistance. Proc. Natl. Acad. Sci., 99:56385642.

8.Jacoby G, Cattoir V, Hooper D, MartínezMartínez L, Nordmann P, Pascual A, Poirel L, and Wang $M$ (2008): qnr gene nomenclature. Antimicrob. Agents Chemother. 52:2297-2299.

9.Wang M, Guo $Q, X u X$, Wang X, Ye X, Wu $S$, Hooper $D C$ and Wang $M$ (2009): New plasmid-mediated quinolone resistance gene, qnrC, found in a clinical isolate of Proteus mirabilis Antimicrob. Agents Chemother. 53:1892-1897.

10.Cavaco L M, Hasman H, Xia S, and Aarestrup F M (2009): qnrD, a novel gene conferring transferable quinolone resistance in Salmonella enterica serovar Kentucky and Bovismorbificans strains of human origin. Antimicrob. Agents Chemother. 53:603-608.

11.Tran $J H$, Jacoby $G A$, Hooper $D C$ $(2005 a)$ : Interaction of the plasmid-encoded quinolone resistance protein Qnr with Escherichia coli DNA gyrase. 49(1):118-25.

12.Tran $J H$, Jacoby $G A$ and Hooper $D C$ $(2005 b)$ : Interaction of the PlasmidEncoded Quinolone Resistance Protein QnrA with Escherichia coli Topoisomerase IV. Antimicrobial Agents And Chemotherapy, 3050-3052.

13.Robicsek A, Strahilevitz. J, Jacoby $G A$, Macielag M, Abbanat D, Bush $K$, and Hooper D C (2006c): Fluoroquinolone modifying enzyme: a novel adaptation of a common aminoglycoside acetyltransferase. Nat. Med. 12:83-88.

14.Wang $M$, Tran $J H$, Jacoby $G A$, Zhang $Y$, Wang $F$, Hooper $D C$ (2003): Plasmidmediated quinolone resistance in clinical isolates of Escherichia coli from Shanghai, China. Antimicrob Agents Chemother, 47:2242-2248.

15.Yamane K, Wachino J, Suzuki S, Kimura $K$, Shibata $N$, Kato $H$, Shibayama $K$, Konda T, and Arakawa $Y$ (2007): New plasmid-mediated fluoroquinolone efflux pump, QepA, found in an Escherichia coli clinical isolate. Antimicrob. Agents Chemother. 51:3354-3360.

16.Jacoby $G$ and Medeiros A (2009): AmpC beta-lactamases. Clin Microbiol Rev, 22(1): 161-182.

17.Mammeri $H$, Nazic $H$, Naas T, Poirel L, $L e^{\prime}$ otard $S$ and Nordmann $P$ (2004): AmpC blactamase in an Escherichia coli clinical isolate confers resistance to expandedspectrum cephalosporins. Antimicrob Agents Chemother, 48:40504053.

18.Philippon A, Arlet $G$ and Jacoby $A J$ (2002): Plasmid-determined AmpC-type blactamases. Antimicrob Agents Chemother, 46:1-11.

19.Poirel L, Heritier C, Spicq $C$ and Nordmann P (2004): In vivo acquisition of high-level resistance to Imipenem in Escherichia coli. J Clin Microbiol, 42:3831-3833.

20.Mammeri $H$, Nordmann P, Berkani $A$ and Eb $F$ (2008): Contribution of extendedspectrum AmpC (ESAC) b-lactamases to carbapenem resistance in Escherichia coli. FEMS Microbiol Lett, 282:238-240.

21. Mulvey M R, Bryce E, Boyd D A, OfnerAgostini $M$, Land $A M$, Simor $A E$ and Paton S (2005): Molecular characterization of cefoxitin-resistant Escherichia coli from Canadian hospitals. Antimicrob. Agents Chemother. 49:358-365. 
22.Tracz. D M, Boyd D A, Hizon R, Bryce E, McGeer A, Ofner-Agostini M, Simor A E, Paton $S$ and Mulvey MR (2007): ampC gene expression in promoter mutants of cefoxitin-resistant Escherichia coli clinical isolattes. FEMS Microbiol. Lett. 27():26.5271.

23.Perez-Perez F J and Hanson N D (2002): Detection of plasmid-mediated AmpC betalactamase genes in clinical isolates by using multiplex PCR. J Clin Microbiol 4():215.32162 .

24.Honore $N$, Nicolas $M H$, and Cole $S T$ (1986): Inducible cephalosporinase production in clinical isolates of Enterofacter cloacae is controlled by a regulatory gene that has been deleted from Eischerichia coli. EMBO J. 5:3709-3714.

25.Ortez H J (2005): Test methods: disk diffusion testing, In, Coyle B. M. (Ed.): Manual of Antimicrobial Susceptibility Testing, American Society for Microbiology, 39-52.

26.Clinical and Laboratory Standards Institute (CLSI) (2011): Performance standards for antimicrobial susceptibility testing: twenty-first informational supplement, CLSI document M100-S21, Wayne, Pennsylvania, USA, 30(15):42-46.

27.Black J A, Moland ES, and Thomson KS (20105): AmpC Disk Test for Detection of Plasmid-Mediated AmpC $\beta$-Lactamases in Enterohacteriaceae Lacking Chromosomal AmpC $B$-Lactamases. Journal Of Clinical Microhiology, 43(7): 3110-3113.

28.Lunn A D, Fàbrega A, Sunchez-Céspedes $J$ and Vila J (2010): Prevalence of mechanisms decreasing quinolonesusceptibility among Salmonella spp. clinical isolates. International Microbiology, 13:15-20.

\section{Cattoir V, Poirel $L$ and Nordmann $P$} (2008): Plasmid-mediated quinolone resistance pump (oepA2 in an Escherichia coli isolate from France. Antimicrob Agents Chemother, 52:3801-3804.
30.Han $C$, Yang $Y$, Wang $M$, Wang $A, L u Q$, $X u X$, Wang $C$, Liu L, Deng $Q$ and Shen $X$ (2010): The prevalence of plasmidmediated quinolone resistance determinants among clinical isolates of ESBI or AmpC producing Escherichia coli from Chinese pediatric Patients. Microbiol Immunol; 54: 123-1280.

31.Ma J, Zeng Z, Chen Z, XUX, Wang X, Deng Y, Lï D, Huang L, Zhang Y, Liu J and Wang $M$ (2009): High Prevalence of Plasmicl-Mediated Quinolone Resistance Determinants gnr, auc(fec)-lb-cr, and gept among

Enterobacteriaceas Isolates from Companion and Food-Producing Animaly Antimicrob. Agents Chemother. 5.3(2):519.

32.Liu J H, Deng Y T, Zeng Z L, Gao J H, Chen L, Arakawa Y and Chen Z L (2008): Co-prevalence of plasmid-mediated quinolone resistance determinants (epA. Qnr, and $A A C\left(6 \_\right.$) $-\mathrm{Ib}-\mathrm{cr}$ among 16S rRNA methylase $\mathrm{Rm} \overline{\mathrm{B}}$ producing Escherichia coli isolates from pigs. Antimicrob. Agents Chemother, 52:2992-29(9)

33.Yue L, Jiang $H X$, Liao X P, Liu J H, LiS $J$, Chen $X Y$, Chen $C X$, LüI H, and Liu Y $H$ (2008): Prevalence of plasmidmediated quinolone resistance ynr genes in poultry and swine clinical isolates of Escherichia coli. Vet. Microbiol.. 132:41.4 42() .

34.Yamane K, Wachino J, Suzuki S and Arakawa Y (2008): Plasmid-mediated qep) A gene among Escherichia coli clinical isolates from Japan. Antimicrob. Agents Chemother, 52:1564-1566.

35.Mammeri H, Van De Loo M, Poirel L, Martinez-Martinez $L$ and Nordmann $P$ (2005): Emergence of plasmid-mediated quinolone resistance in Escherichia coli in Europe. Antimicrob Agents Chemother, 49:71-76.

36. Wu J J, Ko W C, Tsai S H, and Yan JJ (2008): Prevalence of ()ir deleminamis among bloodstream isolates of fischerichio coli and Klebsiella preumoniae in a 
Zag. Vet.J.

Taiwanese hospital 1999-2005. J. 38.Strahilevitz J, Jacoby G A, Hooper D C, Antimicrob. Chemother, 10:1093-1099.

37.Park C H, Robicsek A, Jacoby G A, Sahm $D$ and Hooper $D C$ (2006): Prevalence in the United States of aac(6_)-Ib-cr encoding a ciprofloxacin modifying enzyme. Antimicrob. Agents Chemother, 50:3953395.5 and Robicsek $A$ (2009): Plasmid-Mediated Quinolone Resistance: a Multifaceted Threat, Clinical Microbiology Reviews, 22 (4): 664-689.

\section{الملخص المعبى}

ارتباط مقاومة الكينولون عبر البلازميد لعتر ات الاشريشية القولونية المنتجة للبيتا لاكتاماز

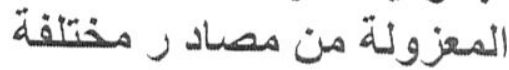

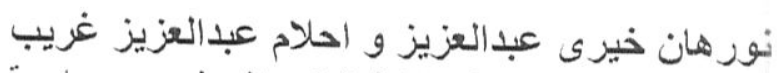

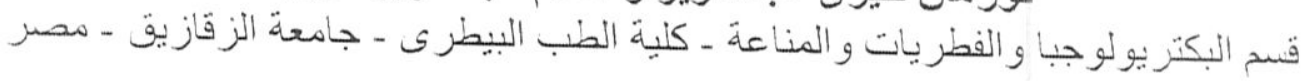

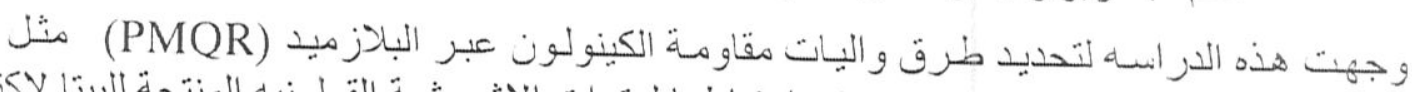

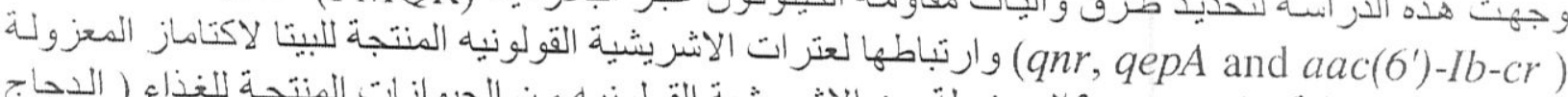

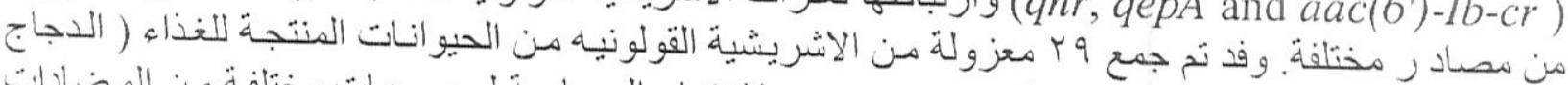

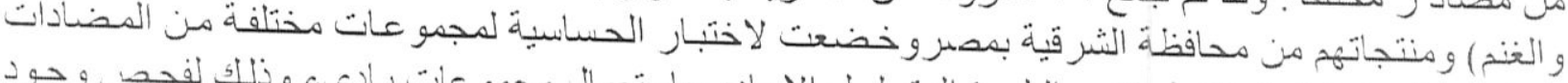

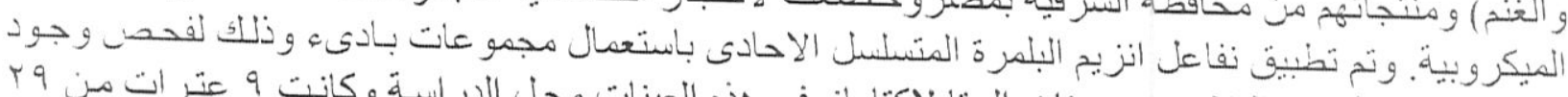

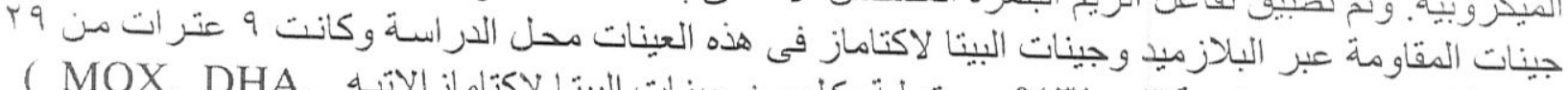

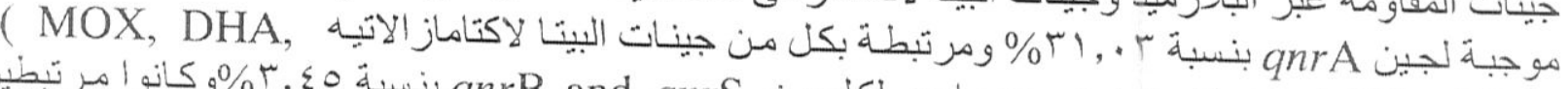

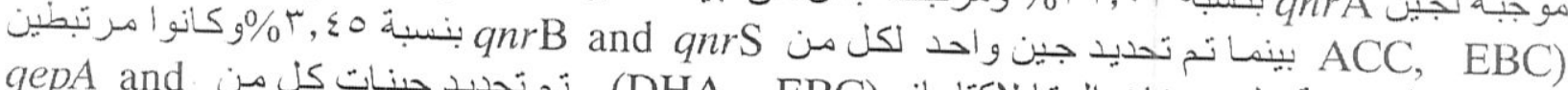

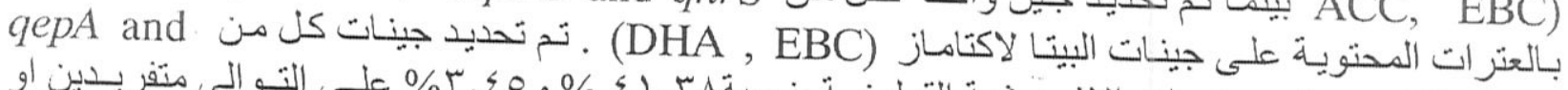
aac(6')-Ib-cr

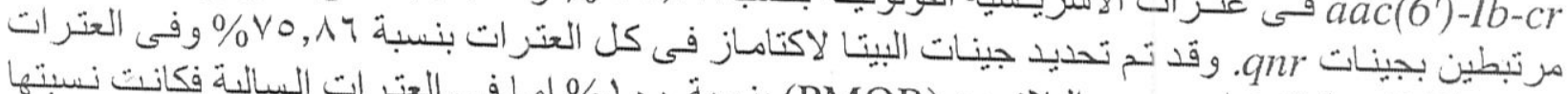

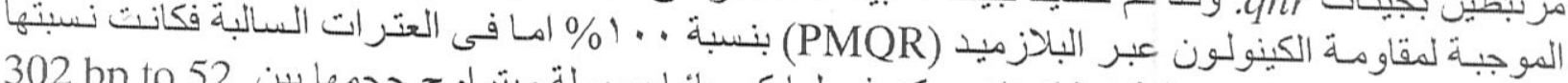

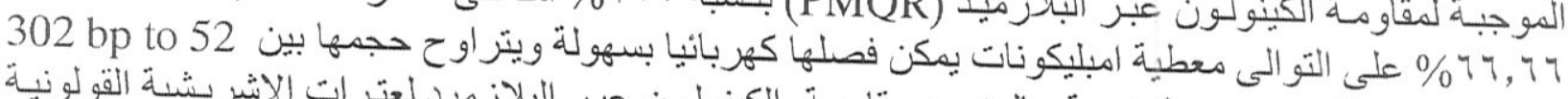

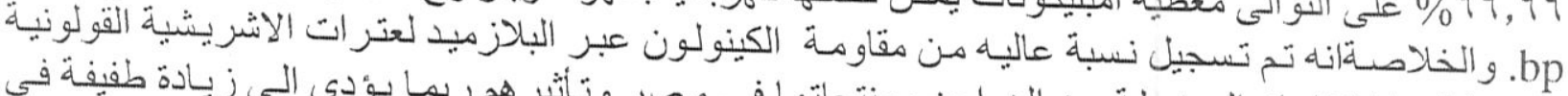

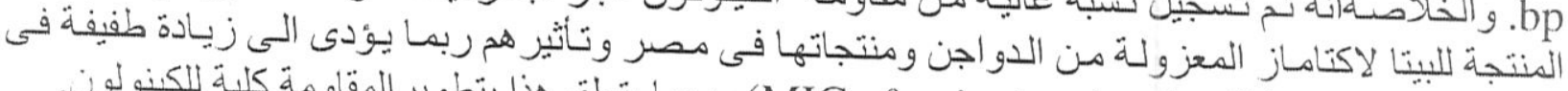
التر كبز الادني المثبط للكينولون (MIC of quinolone) وربما يتعلق هذا بتطوير المقاومة كلبة للكينولون. 